\title{
A Reference Architecture and Model for Sensor Data Warehousing
}

\author{
Simon Dobson, Senior Member, IEEE, Matteo Golfarelli, Simone Graziani, and Stefano Rizzi
}

\begin{abstract}
Sensor data is becoming far more available thanks to the growth in both sensor systems and Internet of Things devices. Much of the value of sensor data comes from examining trends that occur over long timescales, ranging from hours to years. However, making use of data a long time after it has been collected has significant implications for the data-handling systems used to manage it. In particular, the data must be contextualised into the environment in which it was collected to avoid misleading (and potentially dangerous) mis-interpretation. We apply data warehousing techniques to develop an extensible model to capture contextual metadata alongside sensor datasets, and show how this can be used to support the analysis of datasets long after collection. We present our baseline reference framework for sensor context and derive multidimensional schemata representing different modelling and analysis scenarios. Finally, we exercise the model with two case studies.
\end{abstract}

Index Terms-Data Warehouse, Multidimensional Modelling, Sensor Networks, Data Analytics

\section{INTRODUCTION}

$\mathrm{T}$ HE availability of sensor data is projected to mushroom in the coming years, fueled by growth both in "explicit" sensor systems and in the increased "implicit" capabilities of the Internet of Things (IoT). Sensing provides stakeholders with data upon which to base decisions that have increasing importance in managing a huge range of user-centred and societal problems.

Much of the value of sensor data comes from examining the trends and variations that occur over long timescales where "long" may range from hours to years, depending on the context of data collection. This is the point at which sensor data becomes "big data" that can be manipulated using machine learning and other tools from data analytics. The goal of such approaches is to extract further global information from the time series, over and above the local information (in space and time) that may be used operationally. That is to say, the value of a sensor dataset may come both from short-term, tactical, operational use of the information it contains, and also from long-term, strategic uses that permit identification of trends and features that are relevant to future contingency planning and post facto analysis of operations. In many contexts these multiple uses significantly increase the importance and value of any data collected, making the financial case for instrumenting environments stronger: indeed, many sensor deployments are financially viable only when considered from the perspective of years-worth of data collection.

There is, however, a big challenge implicit in this scenario. Making use of data a long time after it has been collected implies a number of things about the data handling

- Simon Dobson is with the School of Computer Science, University of St Andrews, St Andrews, Fife KY16 9SX UK (e-mail: simon.dobson@standrews.ac.uk).

- Matteo Golfarelli, Simone Graziani, and Stefano Rizzi are with DISI, University of Bologna, V. le Risorgimento 2, 40136 Bologna IT (e-mail: matteo.golfarelli@unibo.it,simone.graziani2@unibo.it, stefano.rizzi@unibo.it). system used to manage it. The data must be searchable and findable in order to be retrieved when required; it must be parseable in terms of extracting the data types and ranges used for representation; and -most importantly- it must be contextualised into the environment in which it was collected. By "contextualised" we mean that it must be possible to retrieve the details of the sensors, their installation and operation, as well as simply their data [1].

What particularly makes contextualisation important is the "exposed" lifecycle of a typical sensor. By way of example, consider an air-quality sensor deployed to measure pollutant gases in an industrial setting. The data acquired from this sensor will typically consist of a stream of numbers, and clearly we need to retain the units and range of these data points for later analysis. But the sensor's readings also have metadata such as precision, accuracy, sampling frequency and the like, associated both with the specific transducer being used and the sensing framework controlling it, which must also be recorded if we are to have confidence in analysis. Furthermore the sensor will deteriorate the longer it is in the field, both through mechanical ageing and (in many cases) chemical and physical changes in the transducer itself, both of which lead to decalibration. It may become occluded by plant life, biofilmed by bacterial action, and dirtied by the actions of the very pollutants it is deployed to measure - all of which will affect its behaviour, and so the values in the time series dataset. Cleaning the sensor, whether on an ad hoc basis or according to some schedule, may re-set some of these influences and return the sensor to (some part of) its pristine state.

These contextual changes affect both operational and archival uses of the collected data - but archival data is less amenable to correction. Unless we record at least a large part (and preferably all) of this context, the dataset will become increasingly misleading when analysed. It is important to note that machine learning techniques struggle to address "drift" in sensor values, and so have only a limited ability to address these issues otherwise [2]. 
Similar challenges are well-known in database technology, where the techniques of data warehousing have been developed to ensure that data held for the long term remain robustly analysable in the context of Business Intelligence (BI) applications. However, these techniques have been applied in the context of sensor networking only to a limited extent, and mostly with a small attention to data modelling issues. In this paper we address this omission by developing data warehousing schemata for sensor data. We develop an extensible model that allows for the capture of contextual metadata alongside sensor datasets, and show how this can be used to support the long-term analysis of historical datasets. While our proposal is aimed at long-term data management with storage and explorative analyses in mind, it is fully compatible with the adoption of other problem-specific analytical solutions. Indeed, the proposed architecture and multidimensional schemata are meant to provide a foundation which other analytical modules can rely upon in order to access integrated and consistent data. We argue that such an approach will increase the value that can be extracted from collected data, as well for supporting some increased level of confidence in the strategies and decisions such datasets are used to support.

The remainder of the paper is organized as follows. Section 2 introduces the basics of data warehousing and multidimensional modelling, and the necessary concepts and technologies in sensor data representation. Section 3 presents our baseline reference framework for sensor context, from which Section 4 then derives multidimensional schemata representing different modelling and analysis scenarios. We exercise the model in Section 5 with two case studies, which we then use in Section 6 to draw some conclusions and future directions.

\section{BACKGROUND}

The topic of sensor network modelling has been tackled from many different perspectives. The result is a plethora of approaches whose main goals are either to enable interoperability between processes, or to simplify the analysis of data and the design of applications. In this section we survey the most relevant of these approaches, after first providing some basic notions of data warehousing and multidimensional modelling.

\subsection{Data warehouses and data lakes}

Operational databases are a common feature of information systems, where they enable long-term, language- and application-neutral storage of large volumes of data for running a company's daily business operations. While a goal of database design is to represent the data in a natural, "normal" form, performance and other constraints often dictate part of the data layout. This often involves compromises between meeting different applications' requirements, as well as in issues such as performing data denormalisation for performance reasons. While such compromises are often necessary for production systems, they harm the ability to access data over the long term. The danger is that data that remains uncurated for a long period may become inaccessible, or so inconsistent as to be useless.
Data warehousing is a process for ensuring the longterm viability of data storage and retrieval using a single integrated repository called a Data Warehouse (DW) that is aimed at supporting analysis and decision-making. It starts from the recognition that the schemata appropriate for archival storage may differ from those needed for operational use. Rather than trying to find a compromise between the two, dara warehousing instead develops structured processes for transforming between them. A generic data warehousing architecture normally uses some of the company's operational databases as data sources; while these databases are most often heterogeneous and inconsistent with each other, a DW represents all this data within a single schema. Between the sources and the DW is a process of Extract, Transform, and Load (ETL) which consolidates the multiform data in the operational databases into the uniform structure of the DW. ETL is a periodic and incremental process that also includes data cleansing steps to resolve inconsistencies and remove noise - which may be tolerated in the operational databases but not in the DW.

Modern enterprise architectures are often extended with a data lake, a repository based on low-cost technologies that contains large quantities of raw, unstructured or semistructured data [3]. Intuitively, the data lake is the place for storing, in its native form, current data that is being produced by the various operational processes that make up the enterprise; by contrast, the DW represents the curated set of events that occurred, that can be used by post facto trend analysis, anomaly detection, and other nonoperational processes. This separation implies that, as the enterprise changes, the data lake can be freely reconfigured in support of new processes, with corresponding changes to the ETL process to populate the DW. The challenge is then to manage such changes in a stable way within the DW schema.

\subsection{Multidimensional modelling and OLAP}

The multidimensional model is the foundation for data representation and querying in DW and BI applications [4]. It is based on the observation that the factors affecting decision-making processes are business-specific facts such as sales, hospital admissions, maintenance, and so on. We refer to these collections of facts as cubes, although they may in fact be higher-dimensional. Data is structured as points in an $n$-dimensional space, whose coordinates correspond to categorical dimensions for analysis. Each point represents an event that occurred and is described by a set of (usually numerical) measures relevant to decision-making processes. Normally, each dimension is associated with a hierarchy of aggregation levels that allow events to be grouped in different ways.

In relational implementations of DWs, multidimensional cubes are normally stored using star schemata [4]. A star schema is composed by (i) some dimension tables, one for each hierarchy, each identified by a surrogate key (i.e., a DBMS-generated progressive identifier) and including one column for each level of that hierarchy, and (ii) one fact table, whose primary key is the combination of foreign keys referencing all dimension tables, and including one column for each measure. To improve performance (by reducing 
the number of joins) dimension tables in star schemata are often denormalised (while keeping the rest of the schema normalised).

DW schemata can be designed using any data analysis technique, but specialised techniques also exist that are targeted specifically at multidimensional (as opposed to traditional relational) modelling. In this paper we make use of the Dimensional Fact Model (DFM) [4], which structures the schema in terms of the facts to be analysed, their dimensions, measures, and levels.

The main paradigm for analysing and querying multidimensional cubes is On-Line Analytical Processing (OLAP). An OLAP session consists of a sequence of queries, each obtained from the previous one by applying an OLAP operation [4]. Each query groups measured values by some subset of levels using an aggregation operator (e.g., sum or average), and optionally applies a selection predicate. The main OLAP operations are roll-up, which gives a broader view of a fact by further aggregating data; drill-down, which gives more detail on a fact by disaggregating data; and sliceand-dice, which selects either a slice or a dice of the cube by expressing a selection predicate.

\subsection{Sensor network data modelling}

While DW technologies are part of some proposed sensing architectures, the details on how the data has been modelled are mostly omitted. An example of this can be found in Huo et alia [5], where the authors present a framework for faultdiagnosis that employs an enterprise DW as an integrated repository for monitoring data, but do not explain the specific data model adopted. Scriney et alia [6] proposes a methodology to obtain multidimensional data cubes starting from XML and JSON data sources. The methodology is composed by two main steps: converting an XML or JSON schema definition into a novel graph structure called StarGraph, followed by populating the data cubes with the events coming from the network. The main limitation of this approach is that it works only with XML and JSON data sources that provide a schema definition. Note that the idea of supporting (or even automating) the design of DWs is not new: early approaches date from the 1990s [7], and remains an on-going research effort [8]. However, the issue with these techniques is that they are often tied with specific types of data sources (e.g., XML/JSON for [6], data vaults for [8]).

Among the approaches that focus on the interoperability aspect, the SensorML [9] framework provides a set of XML schemata to describe sensor networks from the physical systems to the measurements and processes involved. De et alia [10] present work aimed at improving the interoperability aspect of IoT. The result is a semantic model approach where the resources of the network are exposed through web services, enabling semantic search and reasoning over both devices and the data they provide. BOnSAI [11] takes a more focused approach by specialising other ontologies (e.g., OWL-S [12]) for ambient intelligence scenarios: once again, the goal is to create a machine-interpretable representation of sensor networks to improve interoperability.

An interesting tool to support the design of applications over sensor networks is SEM [13], which is a framework that represents the sensor network through two different metamodels: a functional metamodel and a data metamodel. The former describes the network as a set of exposed services, while the latter describes the available data sources. Through these two metamodels the application designer can obtain a high-level view of the network to better manage its complexity.

\section{Reference ARChitecture AND domain MODEL}

In this section we present our reference framework, which is composed of a functional architecture (section 3.1) and the domain model (section 3.2). This framework will then be used as a reference for all proposed multidimensional schemata and use cases for the rest of the paper.

\subsection{Functional architecture of the analytical system}

Figure 1 shows the proposed functional architecture that we will use to contextualise the multidimensional schemata presented in section 4 and the use cases presented in section 5 . We start by describing the processes and how they interact, and then proceed to explain how we envision its implementation from a technological perspective.

The architecture shown in figure 1 is separated into two different large-scale areas by the vertical dashed line. On the left there is the sensor network, while on the right there are all the processes that read, elaborate, and store the data coming from that network.

We treat the sensor network as a collection of (typically computationally-constrained) nodes collecting data from the physical environment. The nodes often make use of mesh or other network protocols and use spanning trees for collecting and aggregating data to a small set of "sink" nodes - none of the details of which affect the current work. We simply assume that the data collected is somehow returned to the cloud for processing. (It is worth noting in passing that many sensor networks are actually sensor/actuator networks that need to make local control decisions. Our architecture supports these too, and would probably want to record both the sensed data and the decisions made as a result, for later analysis.)

\section{Users and use cases}

The users of the proposed architecture can be grouped into three main agents:

- A data scientist, who has both high IT and analytical skills and can therefore exploit both the wellstructured and organised data stored in the DW and the less consistent and structured contents of the data lake to carry out her analyses.

- A BI user, who has broad knowledge about the domain of the data but needs accessible analytical BI tools (e.g., Tableau ${ }^{1}$, MicroStrategy ${ }^{2}$, and so forth) to accomplish her goals. The BI user typically only has access to structured and well-organised data stored in the DW.

1. https://www.tableau.com

2. https://www.microstrategy.com 


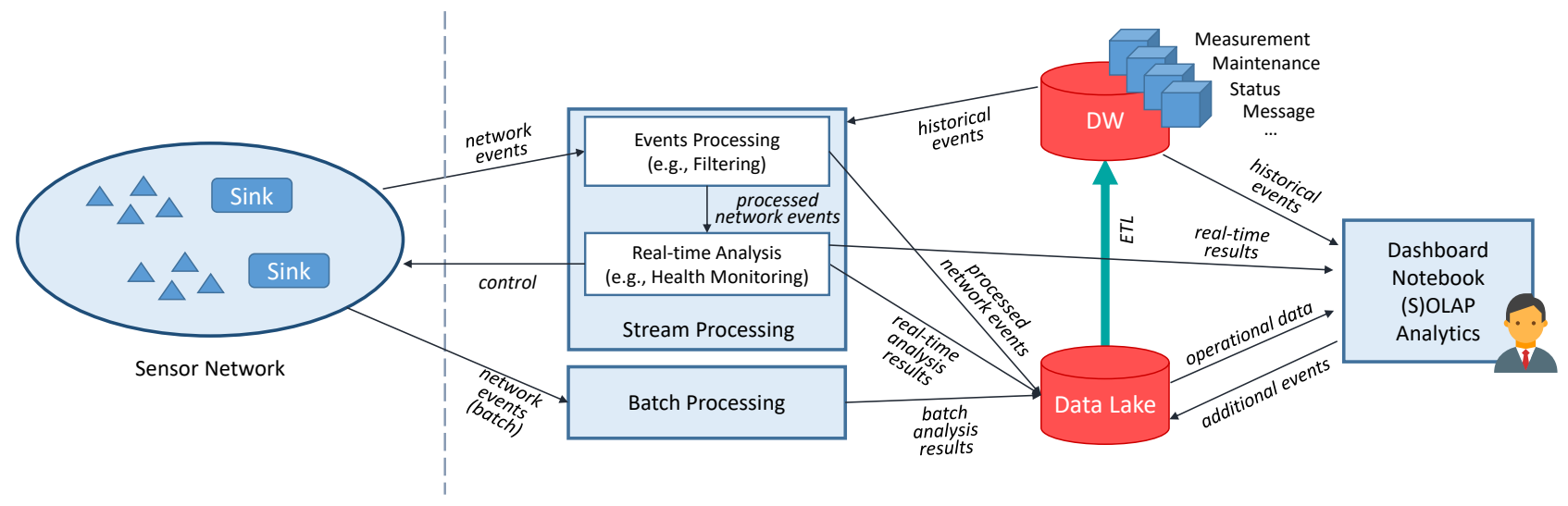

Fig. 1: Functional architecture of the analytical system for sensor networks

- A technician, who is a user who has very specific domain knowledge but lacks IT skills. These users include, for instance, any maintenance worker who might be interested in monitoring in real-time the status of the network.

The interface capabilities required for different users will change depending on the type of final application. Typically, data scientists and skilled domain users prefer having more control on data and more powerful tools (e.g., Notebook tools, OLAP interfaces, and analytics), while domain users prefer simple and focused information such as Key Performance Indicators (KPIs) that can be presented through dashboard tools. We will not discuss in details the wide range of possible applications the proposed architecture can be used for: instead, in the following we will discuss in depth the domain model that is needed to support them.

The typical data flow in the architecture sketched in figure 1 can be described as follows. Every event sent by a device in the sensor network to the analytical system is processed in either a streaming or a batch fashion as appropriate. In the former case the results of the computation can be both directly presented to the end user and also stored in the data lake for future use; in the latter case the results are simply stored in the data lake. When processing events in real time the system might also react and respond to control the network, thus creating a feedback loop. Once the results of the processing have been stored in the data lake, they can be directly accessed by users (data scientists) and applications for further analysis and elaborations. Periodically (which might be hourly, daily, weekly, and so on) the data in the data lake is used to feed the DW through the ETL process. Once in the DW, the events become also available to BI users by means of OLAP analysis tools.

\section{Event processing and storage}

Stream processing is one of the two intermediary processes between the sensor on one end and users and storages on the other. The typical output of the sensor network is considered as a stream of events (e.g., temperature measurements) that must be processed in a timely fashion. In our architecture this process is decomposed into two different subprocesses, events processing and real-time analysis. The former includes simple operations such as filtering and smoothing of the input events, while the latter uses the output of the former and the historical data in the DW to perform more complex (but still stream-based) analytical functions such as forecasting, network monitoring, and so on. We assume that the real-time analysis process can directly interact with the sensor network, for instance for activity regulation purposes.

In certain scenarios it might be either necessary (for example due to the lack of connectivity) or sufficient (because the timeliness constraints are relaxed) to treat the events coming from the sensor network in larger aggregates rather than singly. In these cases the events produced are handled by batch processing rather than by stream processing. By relaxing timeliness constraints it is possible to perform more complex computations that would not be possible in a realtime scenario. For example, signal reliability techniques can be run at this stage, possibly in conjunction with some more advanced event classification processes [14]. Noisy data can be either tagged or dropped [15] - the latter is often preferable as it allows later analysis of the cleaning processes themselves.

Of course, these styles of processing are not mutually exclusive and, indeed, a mix of the two can be employed to have both real-time results through stream processing and higher quality results from batch processing. The results of both stream and batch processing are loaded into the data lake.

\section{Technology choices}

From a technological perspective, this architecture can be implemented as follows. We ignore the implementation of the sensor network other than to assume the creation of a stream of sensed values.

Perhaps the most promising technology for the analytic system is the Hadoop platform ${ }^{3}$, which provides distributed storage and processing capabilities. The choice of a distributed system is almost mandated in many applications, where a traditional centralised approach cannot accommodate the high data volumes that a sensor network might produce. Furthermore, the Hadoop platform is very flexible and can support many other frameworks that are necessary

3. http://hadoop.apache.org 
for some of the tasks described above. For example, example frameworks for streaming processing include Apache Storm ${ }^{4}$, Apache Spark [16], and Apache Flink ${ }^{5}$. Spark and Flink can also take on batch processing tasks.

For storage, the data lake must be able to handle both unstructured and structured data. The simplest and most general solution is accommodating all kinds of unstructured data as simple distributed files in the HDFS filesystem [17] and structured data through either Apache Hive, SparkSQL, or Impala [18]. (An alternative to using HDFS for all unstructured data is that of adopting more specialised storage types, which generally fall into the NoSQL [19] category: we avoid discussing these solutions as they are out of the scope of this work.) Apache Kylin ${ }^{6}$ provides OLAP processing on top of these (and other) storage and processing solutions. In particular, it is the first engine in the Hadoop ecosystem natively optimized for OLAP workload: it fully support multidimensional schemata (see section 4), enables incremental refresh of cubes, and provides an interactive experience even when billions of tuples are involved [20]. Finally, there some commercial platforms (e.g., Azure IoT Suite $^{7}$ ) that boost the construction of IoT solutions. It is worth noting, however, that even in this case, the problem of accurately modelling data has still to be solved.

\subsection{Domain model}

We now briefly describe the main concepts represented in figure 2, which shows the domain model of an analytical system for sensor networks using the notation of a UML class diagram. When feasible, we also use the SensorML framework [9] as a reference and link the described concepts to the corresponding ones in SensorML.

By agent [21] we mean anything that can be viewed as perceiving its environment and that can act upon it. Agents interact by way of messages, either unicast or multicast between them. We distinguish the following types of agent:

Device A Physical Device is any device inherently associated to a physical object. This concept covers both devices with advanced computational capabilities (e.g., a face recognition camera) and devices that have very basic computational capabilities (e.g., a temperature sensor). In the SensorML framework, the Physical Device concept corresponds to the Component and System concepts. While a physical device represents a physical object, a Logical Device is instead a role that a physical device might have at a particular time. For instance, we might have a logical device called Kitchen Temperature Sensor that can be seen as an abstraction of all the physical sensors that, over time, are used to measure the temperature in the kitchen; in this example, substituting a faulty temperature sensor S1 at some time with a new sensor S2 would result in two different instances of Physical Device (S1

\footnotetext{
4. http://storm.apache.org

5. https://flink.apache.org

6. https://kylin.apache.org/

7. https://azure.microsoft.com/en-us/suites/iot-suite/
}

and S2) both corresponding to the same instance of Logical Device, the Kitchen Temperature Sensor. Each logical device can also be a part of another device (described through a selfaggregation in the diagram), which is especially useful when describing complex systems like a smart house, where multiple sensors can coordinate to achieve a common goal. Furthermore, a logical device can be either stationary or mobile: in the former case the device has also a reference location, which is the location where the device has been placed. Finally, the assignment of a physical device to a logical device is represented through the Assigned Device class.

Process All those agents that do not inherently have a counterpart in the physical world. An example of process is a monitoring application that runs in the cloud. In the SensorML framework, the Process concept corresponds to the ProcessModel and ProcessChain concepts.

Person Any human agent that interacts with the environment and other agents. A typical example is a technician that performs maintenance over the sensor network.

A measurement refers to an observation of a property made at a specific time. Usually, but not necessarily, a measurement refers to a specific location, and may be the result of some kind of low-level transformation such as smoothing: this aspect is modelled by the Transformation class. A typical example of measurement is the temperature sensed by a thermometer. Additionally, a measurement is characterised by estimated precision and accuracy, which define how detailed and how reliable that particular measurement is. Adverse environmental conditions, wear-andtear, and other aspects might influence the accuracy of sensors and so (when available) an estimate of the accuracy of the measurement can greatly improve the quality of the analyses. In SensorML, the definition of a measurement is quite loose and is not explicitly modelled: however, the underlying meaning remains the same.

A maintenance operation includes any operation such as calibration or component substitution performed either on a physical device or on a process by another agent. In SensorML, maintenance operations would be included in the general definition of event, which is used to track the history of a device. A status check represents a check performed by one agent over another, over either a process or a physical device. A check can refer to many different types of assessments, for instance, the assessment of the battery level, the confirmation of a malfunctioning device, or even a notification of a device that goes into power-saving mode.

\section{Multidimensional SCHEMATA}

Figure 3 uses the Dimensional Fact Model (DFM) to show the four multidimensional schemata derived from the domain model presented in section 3.2. Boxes represent a fact to be analysed; circles represent its dimensions and levels, with which may have measures included in the lower part; and arcs represent many-to-one relationships to be used for 


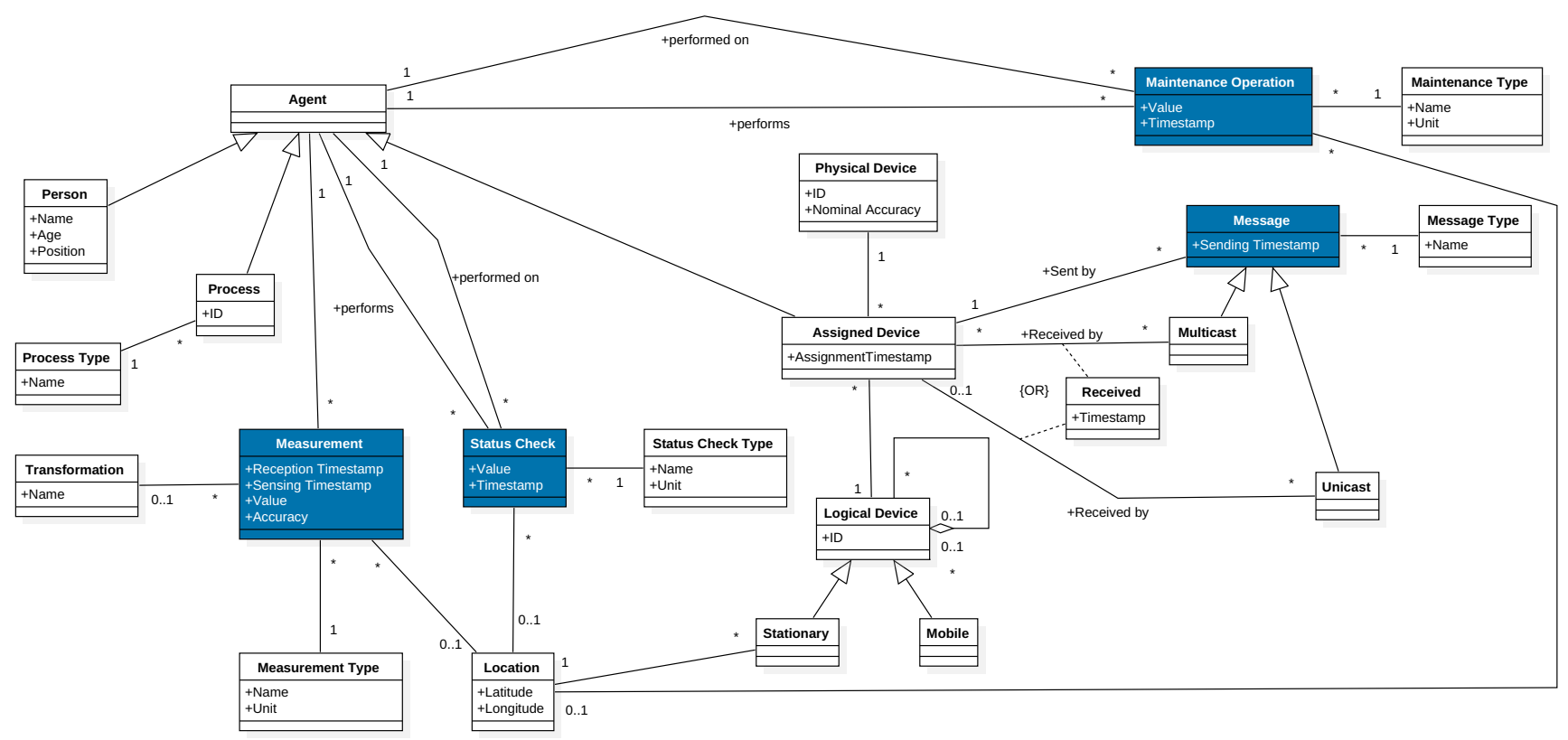

Fig. 2: UML class diagram that describes the domain model of an analytical system for sensor networks

aggregation. Among the concepts represented in the class diagram in figure 2 , those whose dynamic nature makes them suitable to be modelled as facts are Measurement, Maintenance Operation, Status Check, and Message. Before describing these facts one by one, we make a few general observations related to all the schemata:

- The concept of agent is represented as a dimension, and its specialisation into persons, processes, and devices (as of figure 2) is translated in the DFM as an additional level Agent Type, whose domain has values 'Person', 'Process', and 'Device'. The levels that branch from Agent are optional and grouped into three different sets, one for each type of agent. Based on the value of Agent Type, only one group of levels will be meaningful, e.g., if Agent Type = 'Person', then only Age and Position will take a value.

- For devices, the domain model shows that one assigned device is related to one physical and one logical device. So, the agents corresponding to assigned devices can be grouped by either Logical Device or Physical Devices. The recursive aggregation of logical devices is represented in the DFM using a recursive hierarchy (the looping arrow on Logical Device).

- As with the specialisation of Agent, the two different types of logical devices (stationary and mobile) are differentiated through an additional level called Logical Device Type, while the Location level is optional as it is relevant only for stationary devices.

- The Location level also deserves clarification, as it can be used in two different ways. The simple way is that of giving it a simple categorical domain (e.g., \{'Rooftop', 'Control' Room', ...\}) to approximate the geographical positioning of the devices and events. The more complex - but also more expressive - way is ro employ geographical coordinates and geome- tries that can be fully exploited through Spatial OLAP (SOLAP) technologies [22], which allow the user to perform specific OLAP operations tailored for spatiotemporal data and enable location intelligence.

The first schema we discuss is Measurement, shown in figure 3a. Each measurement event is described by two measures and is defined by six dimensions. The two measures are Value and Accuracy, which respectively describe the result of the measurement and its accuracy. Dimension Measure Type, together with the Unit level, defines the context of the measurement. For instance, if Measure Type = 'Temperature' and Unit $=$ 'Celsius Degrees', then the meaning of Value is precisely defined as the temperature in Celsius degrees. The Sensing Time and Reception Time dimensions define the temporal aspects of the measurement. The remaining dimensions define where the measurement has been taken (Location), who took it (Agent), and what kind of processing has been applied to it (Transformation).

The Maintenance and Status Check schemata, shown in figures $3 b$ and $3 c$ respectively, are similar to Measurement. Here the Maintenance Type and Status Check Type levels play the same role that Measurement Type plays in Measurement.

The last schema we discuss is Message, shown in figure $3 \mathrm{~d}$. Unlike from the other facts, messages pertain only to assigned devices, and so the Agent dimension used in all the other schemata has been replaced by Assigned Device. Furthermore, the Message fact has no measures: a message either exists or doesn't. Other modelling choices worth discussing stem from the fact the not all sent messages are actually received, and a communication can be a broadcast where we know who sent the message but not who is supposed to receive it. These two issues are addressed through the addition of the Communication Type and Received levels.

To give some examples that summarise the supported scenarios: 


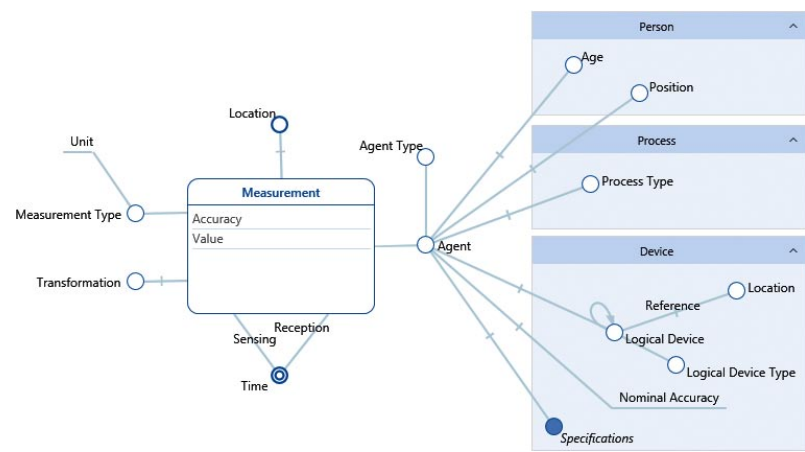

(a)

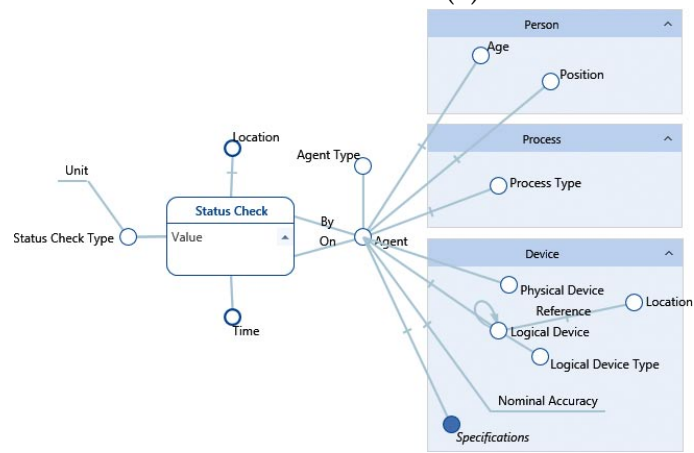

(c)

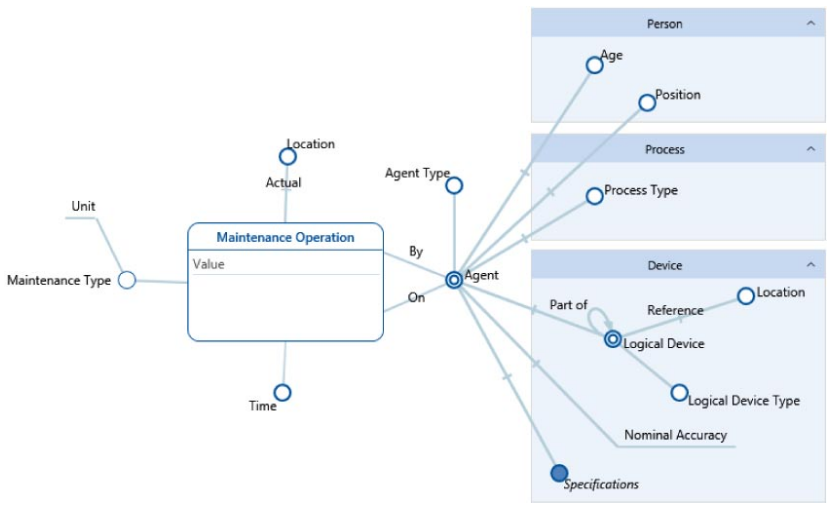

(b)

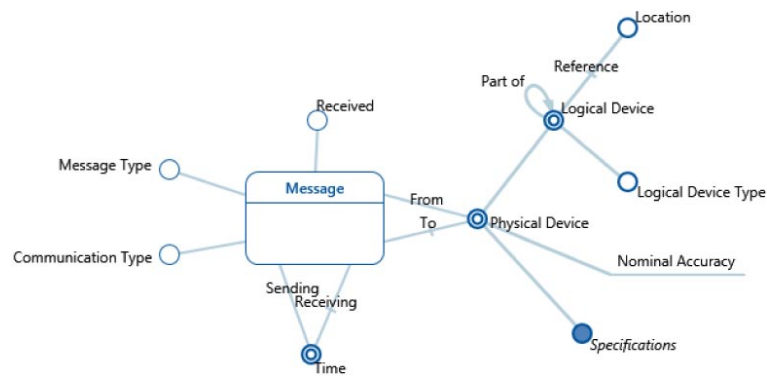

(d)

Fig. 3: Multidimensional schemata for the measurement (a), maintenance (b), status (c), and message (d) events

(i) A successfully delivered message sent from a device $A$ to a device $B$ is represented as an event with Communication Type $=$ 'Unicast', Received = 'True', From Physical Device = 'A', To Physical Device = 'B', with the corresponding sending and receiving times.

(ii) A failed attempt to deliver a message sent from $A$ to $B$ is represented as an event with Communication Type $=$ 'Unicast', Received $=$ 'False', From Physical Device $={ }^{\prime} \mathrm{A}$ ', To Physical Device $={ }^{\prime} \mathrm{B}^{\prime}$, with only the corresponding sending time.

(iii) A broadcast message sent from $A$ and received from $B$ is represented as in the first example but with Communication Type $=$ 'Broadcast'. Each message received is represented as a separate event.

(iv) Finally, a broadcast message sent from $A$ but not received by any other device is represented as a single event with Communication Type = 'Broadcast', From Physical Device $={ }^{\prime} \mathrm{A}^{\prime}$, and the corresponding sending time. In this case the receiving device and time are not specified.

\subsection{Representation and completeness}

For any data warehousing architecture it is important to consider whether it can represent all the data that are anticipated as being collected, and can be extended cleanly to handle unexpected cases - since the goal of the DW is to provide a stable representation unaffected by changes in the operational architecture. How can we provide such assurances for sensor data, where the technology changes rapidly?
We first observe that our domain model captures in many ways the properties of time series and the processes of their collection, rather than sensor datasets per se. Within this broad framework, one may use different measures and levels (as discussed in section 2.2) to handle variations. There is already a substantial body of work that can be applied to identifying and extending these elements. One might, for example make use of SensorML as a data model for the individual sensors. As should be clear from the preceeding discussion, however, SensorML in itself is insufficient for sensor data warehousing, as it excludes many elements of context such as maintenance and does not define either a warehousing process or a query process. It is probably better thought of as a component of the data model together with an exchange format.

For sensor modelling purposes, we can map the main SensorML concepts onto the multidimensional schemata through the Agent attribute. Specifically, the Component and System SensorML concepts correspond to those agents whose Agent Type value is 'Device'. Similarly, the ProcessModel and ProcessChain concepts are represented as agents whose Agent Type value is 'Process'. SensorML also provides a very generic Event concept, which can be used to represent any relevant event related to sensors. However, such generic concepts can hardly be used in analytic contexts without a further modelling step to specialise them into concepts with a more specific semantics (Status Check, Maintenance Operation, etc.).

To describe and enrich the four main concepts that directly model different types of sensors and processes, SensorML includes several other "meta-concepts" such as 
MetadataGroup. While in SensorML these meta-concepts are introduced to facilitate (automatic) processes of resource discovery (which is out of the scope of data warehousing), they can also be useful for analysis purposes. To this end, we used the Specifications attribute in the Agent dimension as a placeholder that should be replaced with the set of descriptive attributes that are relevant to the analysis processes. For example, in the case of sensors for which it is important to know the specific model identifier, one could simply attach to Agent a descriptive attribute named Model that can be used for filtering and descriptive purposes.

From this discussion it is hopefully clear that (i) our modelling approach is broader than SensorML since it also captures aspects not strictly related to sensors, such as mainteinance; (ii) all the concepts directly modelled in SensorML are also modelled in our approach; and (iii) while for several concepts we adopt the same modelling approach of SensorML (either in direct modelling or meta-modelling), in other cases we chose to directly model concepts that are meta-modelled in SensorML, aimed at making a larger number of semantically well-defined concepts available for analyses to BI users.

\section{Case studies}

We now present some examples of how these models can be used in practice to carry out several interesting analyses. We consider the cases of air quality monitoring at an industrial facility (see section 5.1), and of landslide risk management in Italy [23] (see section 5.2). These two examples have been chosen due to their rich and varied requirements, and especially for the need to retain and re-interrogate data over different timescales. In both cases we start by discussing the operational requirements for a sensing system in terms of scientific and business challenges, then we describe a possible suite of sensors to address the problem, and finally we show how the data from this sensor network can be modelled in a DW.

\subsection{Air quality monitoring}

Many large industrial installations operate under tight safeguarding and permission regimes designed to protect civilians near the plants as well as the broader environment. Typically a plant is licensed to emit particular maximum quantities of specific pollutants, and will incur fines or other sanctions for exceeding these limits. Plants will often be required to install systems to monitor their emissions, either directly at point of generation, or indirectly through wider sensing (or both). There may also be further monitoring performed by third-party or regulatory agencies to ensure compliance.

\section{Requirements for air quality monitoring}

The main requirements identified for this scenario are listed in table 1. For each requirement we give a brief description and list the related stakeholders and data sources. There are three main types of stakeholders: the facility, the civilians (who are not directly involved with the facility but who can be affected by its emissions), and the regulatory agencies that have the duty of checking whether or not the facility operates in respect of environmental norms. Each requirement can be satisfied by accessing the data stored in the DW and in the data lake. For those requirements that need access to the DW, we also specify which cubes are needed. Note that, for some requirements (denoted with a star in table 1) we list only the main required cube, however other cubes might be useful to get contextual data: for instance for Requirement (1) it could also be useful to know the status of the network to better assess the accuracy of the measurements.

As well as in the obvious detection of leaks and excess emissions, plant managers, regulators, and other agencies have an interest in the long-term relationship of a plant to its environment. One example would be to detect the buildup of pollutants in the local environment even if the plant were operating as licensed. Another would be to implement a market for pollution with a view to encouraging plants to invest to reduce emissions. These scenarios are covered by Requirement (1) and (2) in table 1. Specifically, the first requirement covers the monitoring of air quality in the long term, while the second covers real-time needs where timely alerts are mandatory (such as in case of leaks). Requirement (1) does not need up-to-date data, so it draws from the DW where data are of higher quality. On the other hand, Requirement (2) cannot afford using stale data, thus it draws directly from the data lake, where measurements are stored in real-time and at the finest level of detail.

One challenge often faced in wider sensing is the problem of attribution: given that a particular situation is observed, who was responsible for it? Suppose residents near a plan wake up one morning to discover a fine white powder coating their cars: what is the powder, where did it come from, and who is to blame? Answering these questions requires a combination of direct analysis (what is the powder?) and potentially the fusion of several data streams to determine possible causes: wind direction may exonerate some plants from consideration, for example, but this requires that such data is available, accessible, and reliable. All these challenges are covered by Requirement (3).

Alongside the challenges strictly related to the management of measurements, the facility also needs to monitor the sensor network itself. Indeed, failures of the network must be detected and addressed as quickly as possible for safety reasons. Moreover, failures imply maintenance costs, which should be kept to a minimum. Requirements (4) and (5) respectively cover the issues related to the (both real-time and off-line) monitoring of the health status of the network and the issues related to the maintenance operations executed to keep the network running.

\section{Sensing air quality}

From the above scientific and business case we can synthesise a set of sensors that we need to deploy in order to address a set of scenarios:

- operational sensing, to ensure that the plant is behaving correctly;

- acute event sensing, to detect unexpected emissions as quickly as possible; and

- contextual sensing, to contextualise the other datasets.

With this in mind, we might decide to install a network of sensors aimed at detecting the possible pollutant 
TABLE 1: Requirements for the air quality scenario

\begin{tabular}{|c|c|c|c|c|}
\hline & Name & Description & Stakeholders & Main Data Sources \\
\hline (1) & Air Quality Measurements & $\begin{array}{l}\text { Quantitatively measure the presence of pollutants in } \\
\text { the air in proximity of the facility }\end{array}$ & Facility, Civilians, Agencies & DW (Measurement)* \\
\hline (2) & Alerts for High Pollution & $\begin{array}{l}\text { Detect in a timely fashion the presence of pollutants } \\
\text { in quantities exceeding safe thresholds }\end{array}$ & Facility, Civilians, Agencies & Data Lake \\
\hline (3) & Environmental Conditions & $\begin{array}{l}\text { Monitor natural phenomena and properties such as } \\
\text { wind, humidity, and temperature in proximity of the } \\
\text { facility }\end{array}$ & Facility, Agencies & DW (Measurement)* \\
\hline (4) & Network Health Status & $\begin{array}{l}\text { Detect potentially malfunctioning parts of the net- } \\
\text { work }\end{array}$ & Facility & Data Lake, DW (Status Check) \\
\hline (5) & Maintenance Operations & $\begin{array}{l}\text { Keep track of the maintenance operations executed } \\
\text { on the network }\end{array}$ & Facility, Agencies & DW (Maintenance Operation) \\
\hline
\end{tabular}

chemicals. Such sensors typically require chemical reactions in their operation, which means that their reagents need to be replaced on a regular basis to avoid exhaustion or decalibration through changing chemistry. This addresses the operational and acute scenarios.

To provide context we might also decide to record environmental data (also known as meteorological or "met" data) such as air temperature and pressure, local wind direction, local humidity, and precipitation. These time series are not needed operationally, but might be crucial in post facto analysis after an incident, where (for example) the chemistry at play might be changed by strong sunlight or an excess of water. They also suggest that we should position sensors differently than we might otherwise, both close to and further from the emission sites (to measure dispersion), and in areas of particular stakeholder interest (wetlands, residential areas, other industrial facilities) to detect potentially dangerous interactions.

\section{Warehousing air quality data}

As noted in table 1, the data needed to satisfy the requirements for this scenario can be drawn from the data lake (for real-time tasks) and from the DW, specifically from cubes Measurement, Status Check, and Maintenance Operation. We focus on the DW and show a practical example of how the conceptual models of figure 3 can be implemented. Figure 4 shows a basic star schema implementation of the multidimensional schemata proposed in section 4 accompanied by a (fictitious) sample of data. Fact tables and dimension tables are denoted by prefixes FT and DT, respectively.

We start by commenting the implementation of the Agent dimension, which is shared by all cubes. Each level (except those not used in this scenario) becomes a column in table DT AGENT. The recursive arc on Logical Device has been ignored here as the topology of the sensor network is quite simple and comprises only one layer of sensors. (In case of more complicated topologies, a parent-child table would be required [4].) Level R. Location is used to identify a generic location but, in more complex networks, it could also be used to refer to specific geographical coordinates. Levels Process Type and Specifications have been omitted as not necessary here. The sample data for DT AGENT represent how both devices and persons can be stored together: of course, for each type of agent, only a subset of levels are relevant and thus filled with meaningful values. The remaining dimensions are quite straightforward, especially the time-related ones and Transformation, which do not need a separate table and can thus be directly included in the fact tables.

For each multidimensional schema we have a fact table, composed by one column for each dimension and one column for each measure. As noted in table 1 and shown in the sample data in figure 4, Requirements (1) and (2) are both supported by the Measurement schema. For instance, the first two events in table FT MEASUREMENT represent pollution-related events (Meas. Type ID =5) computed as the mean of 10 measurements (Transformation = AVG10) made by (logical) devices Indoor $\mathrm{SO}_{2}$ Gas Sensor and Rooftop $\mathrm{SO}_{2}$ Gas Sensor (Agent ID $=\{5,6\}$ ). The other events are all contextual measurements, such as temperature, wind speed, etc. The remaining fact tables are used to store events respectively related to the status of the network and to the maintenance operations.

To close this section, in figure 5 we show a simple example that combines data from different cubes to perform an analysis on pollution levels. The pivot table on top shows the weekly average levels of pollution alongside some weather measurements (average wind speed and temperature). Since during the week from $2017 / 10 / 16$ to $2017 / 10 / 22$ the sensors outside the facility have measured particularly high levels of pollution, the user might decide to drill-down (i.e., zoom in) on this particular week and on a particular sensor placed outside. The bottom pivot table shows the result of the drill-down that disaggregates the measurements of the chosen sensor on a daily basis. Assuming the adoption of a denoising technique, and depending on the adopted data processing policy, noisy measurements could be either tagged or restored. In both cases, the user can determine which data have been affected and evaluate the denoising impact. Furthermore, to check for possible calibration problems, the user also visualises the number of days since the last calibration has been made.

\subsection{Landslides risk management}

Another interesting scenario is the one presented by Giorgetti et alia [23], which describes a wireless sensor network for the monitoring of landslides. The network, deployed on a rockslide in central Italy, gathered both operational data (e.g., communication statistics) and measurements of natural phenomena (e.g., ground movement) in the time period from February to October 2013.

\section{Requirements for landslides risk management}

Table 2 describes the requirements for this case study. The stakeholders are geologists and network engineers. The ge- 


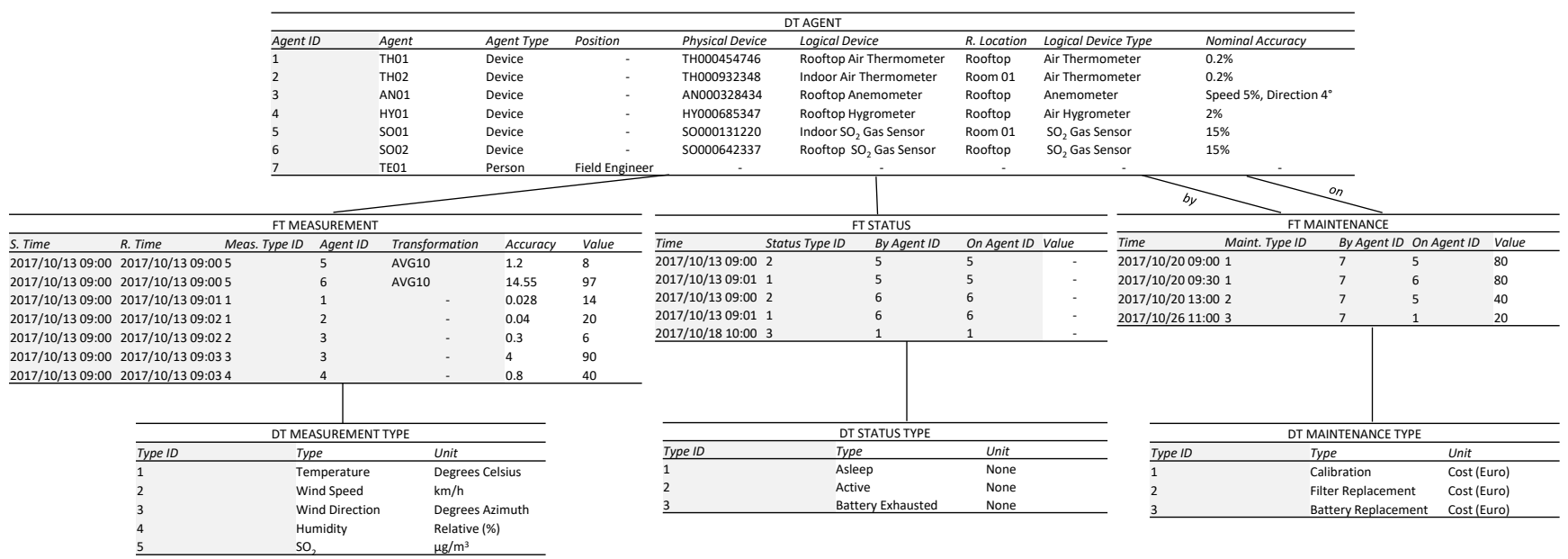

Fig. 4: Star schema and sample data for the air quality scenario

TABLE 2: Requirements for the landslides scenario

\begin{tabular}{|c|c|c|c|c|}
\hline & Name & Description & Stakeholders & Main Data Sources \\
\hline (1) & Ground Movements & Monitor the behaviour of the landslide & Geologists & DW (Measurement)* \\
\hline (2) & Environmental Conditions & $\begin{array}{l}\text { Measure natural phenomena and properties such as wind, } \\
\text { temperature, and humidity }\end{array}$ & Geologists & DW (Measurement)* \\
\hline (3) & Network Synchronization & $\begin{array}{l}\text { Gather statistics on the synchronization among the nodes of } \\
\text { the network }\end{array}$ & Engineers & DW (Status Check) \\
\hline (4) & Communications & Monitor communications between the nodes of the network & Engineers & DW (Message) \\
\hline (5) & Maintenance Operations & $\begin{array}{l}\text { Keep track of the maintenance operations executed on the } \\
\text { network }\end{array}$ & Engineers, Geologists & DW (Maintenance Operation) \\
\hline
\end{tabular}

\begin{tabular}{|c|c|c|c|c|}
\hline \multicolumn{5}{|c|}{ Weekly $\mathrm{SO}_{2}$ Levels } \\
\hline Week & Location & $\mathrm{SO}_{2}$ & Wind $(\mathrm{km} / \mathrm{h})$ & Temperature \\
\hline \multirow{2}{*}{ 2017/10/02 - 2017/10/08 } & Rooftop & 98 & 7 & 13 \\
\hline & Room 01 & 11 & - & 20 \\
\hline \multirow{2}{*}{ 2017/10/09 - 2017/10/15 } & Rooftop & 105 & 9 & 15 \\
\hline & Room 01 & 13 & - & 20 \\
\hline \multirow{2}{*}{$2017 / 10 / 16-2017 / 10 / 22$} & Rooftop & 144 & 4 & 15 \\
\hline & Room 01 & 16 & - & 20 \\
\hline
\end{tabular}

drill-down

\begin{tabular}{lcl}
\hline \multicolumn{3}{c}{ Daily $\mathrm{SO}_{2}$ Levels for Week 2017/10/16 and Rooftop SO, Gas Sensor } \\
\hline Date & $\mathrm{SO}_{2}$ & Days Since Last Calibration \\
\hline $2017 / 10 / 16$ & 135 & 15 \\
$2017 / 10 / 17$ & 170 & 16 \\
$2017 / 10 / 18$ & 192 & 17 \\
$2017 / 10 / 19$ & 205 & 18 \\
$2017 / 10 / 20$ & 106 & 0 \\
$2017 / 10 / 21$ & 102 & 1 \\
$2017 / 10 / 22$ & 98 & 2 \\
\hline
\end{tabular}

Fig. 5: A simple analysis performed on air quality data

ologists are mainly interested in the collected measurements and, to better assess their validity, to the history of maintenance operations. The engineers are mainly interested in the operational data instead, to check if the network is working as intended.

The main goal of this sensor network is to collect data to observe and analyse landslides. To this end, both ground movement data (Requirement (1)) and other environmental measurements (Requirement (2)) must be taken into account. These data can be used to identify relevant factors causing landslides, thus aiding in preventing and reducing their negative impact.

Similarly to the air quality scenario presented in section 5.1, during the monitoring campaign the network it- self was observed to collect operational data. Specifically, the network periodically collected and sent data regarding its synchronization mechanism (Requirement (3)) and the messages exchanged between the nodes (Requirement (4)). These data are crucial to determine if the network is behaving as intended: indeed, the network employs adaptive routing strategies designed to cope with harsh environmental conditions (for example foliage, debris, ground movements, and so forth). Finally, maintenance operations (Requirement (5)) must also be recorded to enable efficient management of the network and reduce costs.

\section{Sensing landslide risk data}

The sensor network deployed for the scenario described above is composed of 15 wireless nodes each equipped with 3 clinometers, 4 wire extensometers, 2 bar extensometers, and 4 soil hygrometers. One of these nodes acts as a sink that sends data to a remote unit for further elaboration. The sink node is also equipped with a weather station for collwectiong met data. Sensor nodes are equipped with lead-acid batteries and a solar cell.

The logical topology of the network is a tree where the root is the sink node. Each node can send data only to its parent, so the sensed data of a node can reach the sink only through child-parent communications. Note that the network is self-organising and has a dynamically defined topology to improve fault tolerance and adaptability with minimal manual intervention.

To communicate, the network adopts a synchronisation mechanism where a given node can be in one of four different phases: association, receive, transmit, and sleep. A node goes in association phase when a it requests to become 


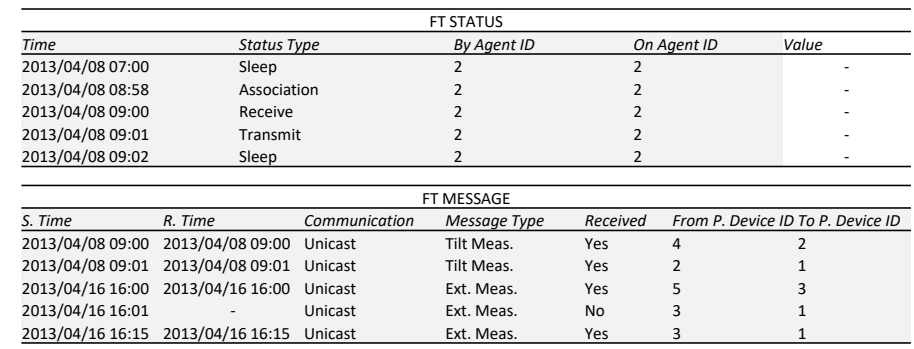

Fig. 6: Star schema and sample data for the landslide risk management scenario

part of the network. The receive and transmit phases are related to the reception and transmission of data, respectively. Finally, to save energy, a node might enter a sleep phase where neither reception nor transmission of data are possible.

\section{Warehousing landslides risk data}

As for the air quality case, figure 6 shows a possible relational implementation with some representative data. We have omitted the implementations of Agent and Measurement since they do not significantly differ from the ones presented in the previous scenario. In this case we focus on facts Message and Status Check, which are used to support Requirement (3) and (4).

The synchronisation mechanism described in the previous section is at the core of Requirement (3). As shown in figure 6 (table FT STATUS), each phase change is recorded as a status check event performed by an agent on itself. The type of phase (sleep, association, receive, and transmit) is represented through the Status Check Type level, while the Time level marks the beginning of the current phase and the end of the previous one. Optionally, the Value measure could be used to explicitly denote the duration of each phase.

As for Requirement (4), each packet exchanged between the nodes of the network can be represented by a message event as shown in figure 6 (table FT MESSAGE). Specifically, the analyses performed in the study presented by Giorgetti et alia [23] involved the computation of two scores, the fraction of packets sent (FPS) and the packet retransmission rate (PRR). The former is computed as the number of packets sent from node $T$ to node $R$, divided by the total number of packets sent by $R$; FPS is thus used to identify which network paths are most (or least) used. The latter score quantifies the quality of a given link between two nodes and is computed as the percentage of retransmitted packets. Both scores can be easily computed based on the data contained in table FT MESSAGE. Indeed, each event represents either an attempt of transmitting a packet (Received $\left.={ }^{\prime} \mathrm{No}^{\prime}\right)$ or a successful transmission (Received $=$ 'Yes'). To compute FPS is sufficient to count the number of successful transmission between all pairs of nodes, while for PRR the relevant events that have to be counted are the failed transmissions.

\section{Conclusions}

We have presented a reference architecture and model for constructing DWs of sensor data. We propose such models as a solution to the challenges posed by the collection and long-term retention and querying of sensor datasets. Without well-founded data handling, we can have no confidence in the conclusions we draw a long time after the data has been collected, which severely limits the applications and analyses we can support. But without such high-value analyses, many sensor network deployments are financially inviable or scientifically questionable. We therefore believe that the use of data warehousing techniques is a crucial component to the evolving landscape of sensor and IoT systems.

We aimed in this paper to cross-fertilise the fields of sensor networks with contributions coming from the data analysis and big data communities. Although the three fields are perceived as quite closed, the requests from both academics and practitioners for a well-defined, robust, and comprehensive architecture and model are frequently apparent. Our experiences in both IoT and "pure" sensing projects show that the adoption of naïve or off-the-shelf solutions reduce analytic capabilities, and such choices, once adopted, are difficult if not impossible to recover from.

Technology for data analysis and big data is evolving fast. Nonetheless the functional architecture proposed as well as the usage of multidimensional cubes are wellestablished, and for this reason we believe that our proposal can be a starting point for a robustm long-term data analysis solution for sensor systems. We intend in future work to validate these ideas more thoroughly against real-world deployments.

\section{Acknowledgements}

The work underlying this paper started as a result of a visit that the third author made to St Andrews in the summer of 2016, and has been partially supported by the UK EPSRC under grant number EP/N007565/1, "Science of Sensor Systems Software".

\section{REFERENCES}

[1] J. Coutaz, J. Crowley, S. Dobson, and D. Garlan, "Context is key," Communications of the ACM, vol. 48, no. 3, pp. 49-53, 2005.

[2] L. Fang and S. Dobson, "Towards data-centric control of sensor networks through Bayesian dynamic linear modelling," in Proc. SASO, 2015.

[3] H. Fang, "Managing data lakes in big data era: What's a data lake and why has it became popular in data management ecosystem," in Proc. CYBER, 2015, pp. 820-824.

[4] M. Golfarelli and S. Rizzi, Data warehouse design: Modern principles and methodologies. McGraw-Hill, Inc., 2009.

[5] Z. Huo, M. Mukherjee, L. Shu, Y. Chen, and Z. Zhou, "Cloudbased data-intensive framework towards fault diagnosis in largescale petrochemical plants," in Proc. IWCMC, 2016, pp. 1080-1085.

[6] M. Scriney, M. F. O'Connor, and M. Roantree, "Generating cubes from smart city web data," in Proc. ACSW, Geelong, Australia, 2017, pp. 49:1-49:8.

[7] M. Golfarelli, D. Maio, and S. Rizzi, "Conceptual design of data warehouses from E/R schemes," in Proc. HICSS, vol. 7. IEEE, 1998, pp. 334-343.

[8] M. Golfarelli, S. Graziani, and S. Rizzi, "Starry vault: Automating multidimensional modeling from data vaults," in Proc. ADBIS, 2016, pp. 137-151.

[9] O. G. Consortium et al., "OGC® SensorML: Model and XML encoding standard," Retrieved from http:/ / www. opengeospatial. org/standards/sensorml, 2014.

[10] S. De, P. Barnaghi, M. Bauer, and S. Meissner, "Service modelling for the internet of things," in Proc. FedCSIS, 2011, pp. 949-955. 
[11] T. G. Stavropoulos, D. Vrakas, D. Vlachava, and N. Bassiliades, "Bonsai: a smart building ontology for ambient intelligence," in Proc. WIMS, 2012, p. 30.

[12] D. Martin, M. Burstein, J. Hobbs, O. Lassila, D. McDermott, S. McIlraith, S. Narayanan, M. Paolucci, B. Parsia, T. Payne, et al., "OWL-S: Semantic markup for web services," W3C member submission, vol. 22, pp. 2007-04, 2004.

[13] F. Cicirelli, G. Fortino, A. Guerrieri, G. Spezzano, and A. Vinci, "Metamodeling of smart environments: from design to implementation," Advanced Engineering Informatics, vol. 33, pp. 274-284, 2017.

[14] J. Ye, L. Fang, and S. Dobson, "Discovery and recognition of unknown activities," in Proceedings of the 2016 ACM International Joint Conference on Pervasive and Ubiquitous Computing (Ubicomp'16): Adjunct, 2016, pp. 783-792. [Online]. Available: doi://10.1145/2968219.2968288

[15] M. L. L. De Faria, C. E. Cugnasca, and J. R. A. Amazonas, "Insights into IoT data and an innovative DWT-based technique to denoise sensor signals," IEEE Sensors Journal, vol. 18, no. 1, pp. 237-247, 2018.

[16] M. Zaharia, R. S. Xin, P. Wendell, T. Das, M. Armbrust, A. Dave, X. Meng, J. Rosen, S. Venkataraman, M. J. Franklin, et al., "Apache spark: A unified engine for big data processing," Communications of the ACM, vol. 59, no. 11, pp. 56-65, 2016.

[17] K. Shvachko, H. Kuang, S. Radia, and R. Chansler, "The hadoop distributed file system," in Proc. MSST, 2010, pp. 1-10.

[18] A. Floratou, U. F. Minhas, and F. Özcan, "SQL-on-Hadoop: Full circle back to shared-nothing database architectures," PVLDB, vol. 7, no. 12, pp. 1295-1306, 2014.

[19] P. J. Sadalage and M. Fowler, NoSQL distilled: a brief guide to the emerging world of polyglot persistence. Pearson Education, 2012.

[20] F. Ming, S. Guannan, and L. Shuaishuai, "Research on multidimensional analysis method of drilling information based on Hadoop," in Proc. ICCC. IEEE, 2017, pp. 2319-2322.

[21] S. Russell, P. Norvig, and A. Intelligence, "A modern approach," Artificial Intelligence, vol. 25, p. 27, 1995.

[22] S. Rivest, Y. Bédard, M.-J. Proulx, M. Nadeau, F. Hubert, and J. Pastor, "SOLAP technology: Merging business intelligence with geospatial technology for interactive spatio-temporal exploration and analysis of data," ISPRS, vol. 60, no. 1, pp. 17-33, 2005.

[23] A. Giorgetti, M. Lucchi, E. Tavelli, M. Barla, G. Gigli, N. Casagli, M. Chiani, and D. Dardari, "A robust wireless sensor network for landslide risk analysis: System design, deployment, and field testing," IEEE Sensors Journal, vol. 16, no. 16, pp. 6374-6386, 2016. 